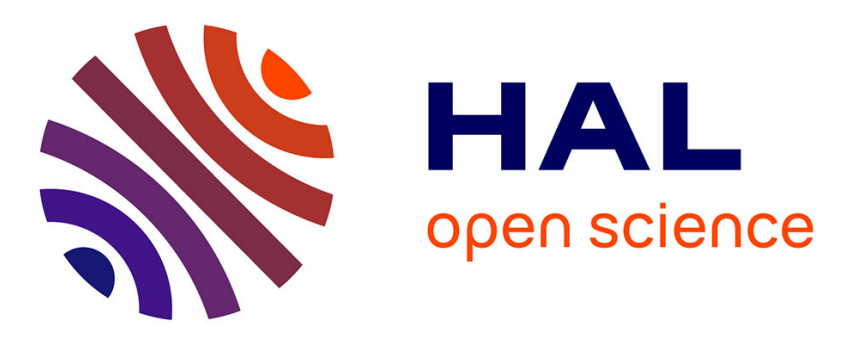

\title{
Fabrication, modeling and integration of a silicon technology force sensor in a piezoelectric micro-manipulator.
}

Ricardo Pérez, Nicolas Chaillet, Krzysztof Domanski, Pawel Janus, Piotr Grabiec

\section{To cite this version:}

Ricardo Pérez, Nicolas Chaillet, Krzysztof Domanski, Pawel Janus, Piotr Grabiec. Fabrication, modeling and integration of a silicon technology force sensor in a piezoelectric micro-manipulator.. Sensors and Actuators A: Physical , 2006, 128, pp.367-375. 10.1016/j.sna.2006.01.042 . hal-00263937

\section{HAL Id: hal-00263937 https://hal.science/hal-00263937}

Submitted on 13 Mar 2008

HAL is a multi-disciplinary open access archive for the deposit and dissemination of scientific research documents, whether they are published or not. The documents may come from teaching and research institutions in France or abroad, or from public or private research centers.
L'archive ouverte pluridisciplinaire HAL, est destinée au dépôt et à la diffusion de documents scientifiques de niveau recherche, publiés ou non, émanant des établissements d'enseignement et de recherche français ou étrangers, des laboratoires publics ou privés. 


\title{
Fabrication, Modelling and Integration of a Silicon Technology Force Sensor in a Piezoelectric Micromanipulator
}

\author{
Ricardo Pérez and Nicolas Chaillet \\ Laboratoire d'Automatique de Besançon - LAB \\ UMR CNRS 6596 - ENSMM/UFC \\ 25, Rue Alain Savary \\ 25000 Besançon, FRANCE \\ Krzysztof Domanski, Pawel Janus and Piotr Grabiec \\ Institute of Electron Technology - ITE \\ Al. Lotników 32/46 \\ 02-668 Warszawa, POLAND
}

\begin{abstract}
This work presents the results of the integration of a force sensor fabricated with the silicon technology in a microgripper actuated by a piezoactuator system. The force sensor consists of a silicon box with an output beam. Forces acting of the beam are sensed in the clamping where the stress is maximum. The voltage read out is made by a Wheatstone bridge. Scaling of force sensors has been done using analytical and numerical tools. Piezoresistivity in the silicon sensor permits measurements of forces of several tens of $\mathrm{mN}$ with a resolution of $10 \mu \mathrm{N}$. Characterisation of sensors in the laboratory has shown the range of deflections obtained for an input force. The former sensor has been later integrated in a piezoelectric micromanipulator. The aim of this integration has been the characterization of the sensor in this new configuration and measurements of handling forces during the manipulation of micro samples in SEM and standard laboratory conditions. Results show that a force range from some $\mathrm{mN}$ to some hundreds of $\mathrm{mN}$ is measured for a sensing micromanipulator.
\end{abstract}

Index Terms-Force sensors, Micro-manipulator, silicon technology, piezoactuator, precision engineering, modelling, micromanipulation, micromachining

\section{INTRODUCTION}

Gorce SEnsors are the more and more demanded devices in many fields. This is the case of the manipulation - of micro samples. There is a real necessity to know the handling forces during manipulation for several reasons. Forces to be applied to handle the object have to be known. If forces go further this value, the sample itself can be damaged. A force sensor would permit to avoid this problem and make the system much more efficient for manipulation tasks. In addition, a control strategy applied to micromanipulation tasks would need information about the handling forces.

Silicon technology for sensing tasks is well known. Performance of silicon sensors is very high [1]. Gauge factor for this kind of technology is 50 and the sensibility $\Delta R / R$ is $0.01 \%$. The gauge factor for semiconductors $\mathrm{n}$ or $\mathrm{p}$ is much more important compared to other technologies such as metallic or serigraphy made gauges. Moreover, this technology is not expensive and is well known. Consequently its feasibility seems to be reasonable for applications in the microhandling.

This work presents the modelling, fabrication and characterisation of a silicon sensor and its integration on a micromanipulator based on the piezoelectric effect. Section II handles with the modelling, fabrication and characterization of the silicon sensor. Section III presents the micromanipulator called MOC (Microrobot On Chip). The integration of the silicon sensor on the MOC for sensing tasks during micromanipulation is presented in section IV. Conclusions will be presented in section V.

\section{SILICON FORCE SENSORS}

The force sensor consists of a silicon cantilever beam with force sensing elements located closed to the clamping. The piezoresistive circuit contains a set of piezoresistors in Wheatstone bridge setup with additional compensation of temperature and offset voltage. Force sensors with different cantilever length (1mm to $3 \mathrm{~mm}$ ) and thickness (up to $100 \mu \mathrm{m}$ ) have been developed to cover a wide area of gripping forces. The end of the cantilever beam is used in this work as an end-effector. Moreover, optional surface structuring of the gripping area of the cantilever is envisaged to improve localization and handling of samples. 
A modification of a double sided silicon micromachining process developed for manufacturing of piezoresistive AFM microprobes has been used to fabricate the force sensors. The fabrication process is schematically illustrated in Fig. 1. Fabrication process has started with both side polished $\langle 100\rangle$ - oriented, $n$ type, $1-5 \Omega . \mathrm{cm}$ silicon wafers. After initial cleaning $600 \mathrm{~nm}$ of thermal oxide was grown and future gripping tips area was defined using standard photolithography (Fig. 1a). Next, using anisotropic $\mathrm{KOH}$ etching, set of silicon tips was fabricated (Fig. 1b). During a standard complementary metal- oxide-semiconductor processing sequence a piezoresistive circuit was fabricated at the support of the future cantilever. Boron and phosphorous diffusions created $p^{+}$-type and $n^{+}$-type regions, serving as connecting diffusion paths and contacts to the substrate, respectively (Fig. 1c). Then, the oxide mask was removed and wafers were oxidized to create clean and thin oxide required for piezoresistors. A set of piezoresistors in a Wheatstone bridge configuration were made through boron implantation and post-implantation annealing (Fig. 1d, e). The back side photolithography of the oxide layer followed by deep plasma etching was performed to create mounting cavities (Fig. 1f). Next, back side photolithography of the nitride layer with a corner compensated pattern and deep, anisotropic silicon wet etching in $\mathrm{KOH}$ solution was used to create a 15-20 $\mu \mathrm{m}$ thick membrane (Fig. 1g). Front side of the wafer was protected form etching with nitride layer. In the next step, the front side photolithography followed by deep, anisotropic silicon wet etching in $\mathrm{KOH}$ solution was carried out to create depression for mounting pads (Fig. $1 \mathrm{~h}$, i). After deposition and photolithography of metal layer (Fig $1 \mathrm{j}$ ) the cantilever shape was defined in the membrane by the last photolithography step and dry plasma etching of silicon (Fig 1k). In the last step the photoresist mask was removed and the device was separated from the wafer (Fig 11).

An exemplary sensor is shown in fig. 2a. The set of 60 blunt tips formed on the end of the cantilever to improve gripping of an object is presented in fig. $2 \mathrm{~b}$. A deep cavity formed in the backside of the sensor body to assure the precise and repeatable positioning and gluing sensor to the gripper is shown in fig. 2c. The depression for contact pads to hide the bonding wires and the epoxy resin protecting the bonding area is presented in fig. $2 \mathrm{~d}$.

\section{B. Modelling}

Modelling of the above proposed force sensor has been necessary to scale correctly the silicon structure to the geometrical and force constraints of the micromanipulator. The silicon beam can be easily modeled considering the mechanics of solids. Deflexion $\delta$ at the tip of the beam is given by equation (1) [2]. Ratio d/F depends upon the geometry and the Young's modulus. Figure 2 shows the dependence of the former on the length and thickness for a given silicon beam of $300 \mu \mathrm{m}$ width. A good trade off to scale the force sensors is a high ratio $\mathrm{L} / \mathrm{t}$. Long and thin silicon beams produce a higher ratio $\delta / \mathrm{F}$. This is particularly important when measuring the sensing force during manipulation.

$$
\delta=\frac{6 L^{3}}{E w t^{3}} F
$$

After scaling the silicon beam, it is necessary to relate the measuring variables to the strain and stress in the beam. From the physics of the piezoresistive sensors, a relationship between the change of resistance and the strain is known and given in equation (2), where R is the resistance and GF the gauge factor of the piezoresistive sensor [3].

$$
\frac{\Delta R}{R}=\varepsilon G F
$$

The Hook's law gives the value of the local stress $\sigma_{\mathrm{x}}$ as a function of the strain $\varepsilon_{x}$. It is possible then combining equations (2) and (3) to calculate the resulting stress. Where $L$ is the length, $w$ is the width and $t$ the thickness of the silicon beam, $E$ is the Young's modulus, $F$ is the applied force, $\sigma_{x}$ the stress and $\varepsilon_{x}$ the strain in the longitudinal direction ( $x$ axis).Depending on the design and location of the gauge, the stress can be longitudinal or transversal (equations (3) and (4) respectively).

$$
\begin{aligned}
\sigma_{x} & =E \varepsilon_{x} \\
\sigma_{y} & =E \varepsilon_{y}
\end{aligned}
$$

If parameters of the piezoresistor such as $R$ and $G F$ are known, it is possible to calculate the strain $\varepsilon_{i}$ and consequently stress $\sigma_{i}$ from equation (3) and (2). Piezoresistive gauges are supposed to be located close to the clamping where the stress is maximum (see figure 1). From the experimental point of view, the mechanical stress $\sigma_{x}$ can be deduced from the variation of the resistance $\Delta R$. Nevertheless, there is no direct relation ship between the stress and the external applied force. An experimental set of measurements is necessary tot determine the correlation between the strain and the applied external force. This can be carried out for instance with a stack piezoactuator as it will be shown in section C. 


\section{Characterisation}

Force sensor characterization is based on the measurements of the offset voltage of the Wheatstone bridge as a function of the cantilever deflection. A piezoceramic actuating element was used for precise bending of the cantilever in the range of $90 \mu \mathrm{m}$ (with a resolution of $2 \mathrm{~nm}$ ). While evaluation of bending force can be done using simplified (one-dimensional) analytical model of a beam, more detailed calculations, taking into account complex 3D shape of the sensor, were performed here using computer simulations. Besides, the additional measurements of the resonance frequencies of the sensors have been made. These values cover the range from $4.481 \mathrm{kHz}$ to 118.1 $\mathrm{kHz}$ for different types of cantilevers. The results of measurements were used to calculate precise values of the thickness of the cantilevers.

It was found that short $(1 \mathrm{~mm})$ and thick $(100 \mu \mathrm{m})$ cantilevers enable measurement of large forces up to $1 \mathrm{~N}$ (for deflection of $40 \mu \mathrm{m})$. The value of the mechanical stress appearing at the base of the cantilever for such force approaches $3 \mathrm{GPa}$, which is still below the critical value of the stress in silicon [3]. Nevertheless, the force sensitivity estimated for long $(3 \mathrm{~mm})$ and thin $(30 \mu \mathrm{m})$ cantilevers was in the range of $100 \mathrm{mV} / \mathrm{mN}$ and was over 50 timer higher than value estimated for the shortest and thickest cantilevers.

It should be noted that the limitation of the force measurement range for sensors based on cantilever may result from the maximum mechanical stress. Even if the maximum mechanical stress appearing in long and thin cantilever bent by force of $5 \mathrm{mN}$ is far below the critical value, the deflexion of the cantilever exceeds $250 \mu \mathrm{m}$. Such a big deflexion is not suitable for gripping of small objects with the cantilever working as an end-effector.

\section{MICRO-GRIPPER (MOC)}

A simplified idea of a micromanipulator system is shown in Figure 3. The micromanipulator called hereafter MOC (MicroRobot on Chip) consists of an actuation system which is piezoelectric and end-effectors assembled to the former. The piezoactuator system is composed by two piezoceramic parallel bimorphs machined by the ultrasonic technique. Each bimorph piezoactuator has 2 DoF (Degree of Freedom). The end-effectors used to handle the sample are Ni made and machined using the LIGA technique.

Assembly of the piezoactuator system and end-effectors results in a MOC micromanipulator packaging shown in figure 2. Both versions are very compact and flexible. This means that these configurations can be easily integrated in much more complex systems such as micromanipulation platforms. The performance of the MOC micromanipulator is listed in table 1. Output displacement $\delta$ per finger is \pm 200 microns out the xy plane and \pm 80 microns in the xy plane at $\pm 100 \mathrm{~V}$ which gives a total opening/closing motion of 320 microns. First resonance frequency is at $400 \mathrm{~Hz}$ in the $\mathrm{z}$ axis and $1 \mathrm{kHz}$ in the $\mathrm{y}$ axis.

\section{A. Modelling}

The model proposed by Smits [4] is applied to bending piezoactuators working outside the plane of the electrodes (z axis). An extension of this model has been first proposed by De Lit et al [2] to take into account the displacement in the $x y$ plane. The resulting work principle is shown in Figure 5. Case a shows the bending displacement in the $\mathrm{z}$ axis, case $\mathrm{b}$ corresponds to the displacement in the $x y$ plane and finally $\mathrm{c}$ shows the coupled displacement of $a$ and $b$. The resulting equations for the working principle proposed above can be written in a compact matrix form with coefficients $a_{i j}$ as follows according to De Lit [5].

Coefficients for both working modes, called hereafter y- and z-modes, noted as $a_{i j}$, depend upon the geometry and material coefficients: length $L_{P Z T}$, width $w$, thickness $h$, compliance $s_{11}$, piezoelectric charge coefficient $d_{31}$ and permittivity $\varepsilon_{33}$. The deflexion angle $\alpha$, deflexion $\delta$ can be calculated when a moment $M$, force $F$, pressure $p$ or voltage $V$ is applied. A further extension of this model has been done in order to take into account the endeffectors of length $L_{E E}$. A simplified model of the piezoceramic beam plus end-effector is shown in Figure 5 . When a force $F$ is applied on the tip of the end-effector due to the micromanipulation, a moment $M$ is generated and the resulting deflexion will depend upon this force. In this model, the end-effector is supposed stiff and the small angles theory is applied. Equations describing the deflexion of geometry shown in Figure 6 under no forces are listed below for $\mathrm{y}$ - and $\mathrm{z}$-mode.

$$
\begin{aligned}
& \delta_{y}(x)=\left\{\begin{array}{l}
0<x \leq L_{P Z T}, \quad \delta_{y}^{P Z T}(x) \\
L_{P Z T}<x \leq L_{E E}, \quad \delta_{y}^{P Z T}\left(L_{P Z T}\right)+\alpha_{y}^{P Z T}\left(L_{E E}\right) \cdot x
\end{array}\right. \\
& \delta(x)=\left\{\begin{array}{l}
0<x \leq L_{P Z T}, \quad \delta_{z}^{P Z T}(x) \\
L_{P Z T}<x \leq L_{E E}, \quad \delta_{z}^{P Z T}\left(L_{P Z T}\right)+\alpha_{z}^{P Z T}\left(L_{E E}\right) \cdot x
\end{array}\right.
\end{aligned}
$$

Where $\delta_{i}^{p z T}$ and $\alpha_{i}^{p z T}$ can be calculated from the following equations according to Smits [4] and De Lit [5] models respectively: 
$\delta_{y}^{P Z T}=-\frac{3 d_{31} V_{y}}{4 h w} x^{2}(4)$ and $\delta_{z}^{P Z T}=-\frac{3 d_{31} V_{z}}{4 h^{2}} x^{2}$
$3 d V$

$\alpha_{y}^{P Z T}=-\frac{3 d_{31} V_{y}}{4 h w} x \quad(6)$ and $\quad \alpha_{z}^{P Z T}=-\frac{3 d_{31} V_{z}}{4 h^{2}} x$

When a force $F$ is applied on the end-effector, displacement $\delta$ becomes:

$\delta_{y}^{F}(x)= \begin{cases}0<x \leq L_{P Z T}, & \delta_{y}^{P Z T, F}(x) \\ L_{P Z T}<x \leq L_{E E}, & \delta_{y}^{P Z T, F}\left(L_{P Z T}\right)+\alpha_{y}^{P Z T, F}\left(L_{P Z T}\right) \cdot x+\delta_{y}^{E E, F}(x)\end{cases}$
$\delta_{z}^{F}(x)= \begin{cases}0<x \leq L_{P Z T}, & \delta_{z}^{P Z T, F}(x) \\ L_{P Z T}<x \leq L_{E E}, & \delta_{z}^{P Z T, F}\left(L_{P Z T}\right)+\alpha_{z}^{P Z T, F}\left(L_{P Z T}\right) \cdot x+\delta_{z}^{E E, F}(x)\end{cases}$

In this case, flexion $\delta_{i}^{F}$ of the piezoceramic beam is due to the voltage and the bending moment as shown in the following equations according to figure 6.

$\begin{aligned} \delta_{z}^{P T T, F} & =-\frac{3 d_{31} V_{y}}{4 h w} x^{2}+\frac{6 s_{11}^{E} M_{z}}{h w^{3}} x \\ \delta_{z}^{P T T, F} & =-\frac{3 d_{31} V_{z}}{4 h^{2}} x^{2}+\frac{3 s_{11}^{E} M_{y}}{4 w h^{3}} x\end{aligned}$

The resulting output angle $\alpha$ after applying force $F$ is calculated then from the general model as shown in the following equations:

$\alpha_{y}^{P Z T, F}=-\frac{3 d_{31} L_{P Z T}^{2}}{2 h w} V+\frac{12 L_{P Z T}}{h w^{3}} M_{z}$

$\alpha_{z}^{P 2 T, F}=-\frac{3 d_{31} L_{P Z T}^{2}}{2 h^{2}} V+\frac{3 s_{11}^{E} L_{P Z T}}{2 w h^{3}} M_{y}$

A FE model (Ansys) has been applied to model the micromanipulator model shown in Figure 6. Results of the former have been compared to those of the analytical model. Figures 6 and 7 show the results of the analytical model (line) and FE tool (dots). Agreement is quite good for small forces of several tens of mN. Nevertheless, the agreement is less good for forces close to the blocking force. Shifting between both models can be justified by the fact that the boundary conditions of the contact interface between the piezoceramic beam and the endeffector have been simplified in the analytical model. For large forces such as those close to the blocking forces, the analytical model is less accurate due to its simplicity.

\section{B. $\quad$ Fabrication and characterisation}

Fabrication of the micromanipulator consists of basically three main steps. 1. Fabrication and machining of the piezoceramic system, 2. Assembly of the end-effectors and 3. Electric connections of the whole system. Step 1 is partially carried out in clean room. Electrodes of the piezoceramic are structured by photolithography. Machining of the piezoactuator system is made using the ultrasonic technique. End-effectors are made by LIGA technique and are assembled to the tips of the piezoactuator system.

The performance of the MOC micromanipulator is listed in table 1. Output displacement per finger is \pm 200 microns out the $x y$ plane and \pm 80 microns in the xy plane at $\pm 100 \mathrm{~V}$ which gives a total opening/closing motion of 320 microns. First resonance frequency is at $400 \mathrm{~Hz}$ in the $\mathrm{z}$ axis and $1 \mathrm{kHz}$ in the $y$ axis. Hysteresis loops are compared with the displacement calculated with the analytical moment presented in the previous section. Hysteresis reaches $25 \%$ in open loop operation. This value can be reduced to less than $5 \%$ applying a convenient open-loop control strategy [6].

\section{INTEGRATION OF A FORCE SENSOR IN THE MICRO-GRIPPER}

In order to validate the concept of the force sensor based on silicon technology, the former has been integrated in the structure of the piezoceramic actuator. Figure 8 shows the piezoceramic structure working as piezoactuator and the silicon force sensor to be integrated on the tip. The resulting sample can be actuated by applying a voltage and can also be able of sensing the applied forces. The silicon force sensor is assembled by gluing on the tip of the piezoelectric fingers of the micromanipulator (see figure 8). Due to the assembly operation some misalign can result between the force sensor and the piezoceramic finger. The former has to be avoided as much as possible in order to be close to the theoretical case in which the force is applied perpendicular to the tip of the end-effector. The output signal from the sensor in Ohms $(\Delta \mathrm{R})$ gives the variation of the resistance and consequently the applied force according to equations 2 to 4.

\section{A. Characterisation}

The characterisation of the integrated silicon force sensor in the micromanipulator has been done under two different boundary conditions. Figure 9 shows the set up used to measure the variation of the resistance as a 
function of the displacement measured in the laboratory. The whole structure, piezoactuator and silicon force sensor can be shifted by using a micrometric $x-y$ stage. The resistance of the sensor keeps constant until the beam touches the support. When the beam touches the support in figure 8 , a change of the resistance $\Delta \mathrm{R}$ in the sensor takes place. The set-up used for the characterization of the implemented force sensor on the piezoceramic beam is shown in figure 10 .

The ratio $\Delta \mathrm{R} / \mathrm{R}$ in $\%$ versus the displacement of the silicon beam is shown in figure 9 (left). Geometrical and material parameters are known and consequently force applied on the beam can be deduced from equation (1). The applied force calculated from equation (1) is shown in figure 9 (right). Results in both figures show the linearity of the silicon sensor with a beam of $2.5 \mathrm{~mm}$ length, $102 \mu \mathrm{m}$ thickness and $344 \mu \mathrm{m}$ width. Similar results have been obtained with sensors fabricated under the same conditions.

Due to the environmental effects on the value of the resistance, the nominal value for each resistance has to be measured at the beginning of any whether characterization or manipulation. These values can differ from those obtained in the laboratory for the calibration of the single sensors. After measuring the nominal value of the resistance before the measurements of $\Delta \mathrm{R}$, the general trend is linear and as expected the values of forces realistic with what is expected for the configuration shown in figure 12.

\section{CONCLUSIONS}

A silicon force sensor has been integrated in a micromanipulator actuated by piezoelectric effect. Characterisation of the single force sensor has shown that forces from some $\mathrm{mN}$ to several hundreds of $\mathrm{mN}$ can be measured. The silicon sensors have been next integrated on the tips of a piezoceramic structure used as a micromanipulator. The performance of the sensor has been then characterized in laboratory. Results obtained show a good linearity with a theoretical force resolution going from some tens of $\mu \mathrm{N}$ to some mN. The low cost and good knowledge about silicon technology, force silicon sensors seem to be an attractive solution for sensing problems in micromanipulators. One of the most difficult points in this approach is the assembly and technological problems related to the set up, such as bonding of wires from the silicon sensor.

\section{REFERENCES}

[1] Bau, H. H., de Rooij, N. F. and Kloeck, B. Sensors a Comprehensive Survey. Vol. 7.VCH. 1994.

[2] Gere, J. M. and Timoshenko, S. P. Mechanics of materials.

[3] Petersen K., Silicon as a mechanical material, Proc. of the IEEE, Vol. 70, No 5, May 1982, pp. 420-45

[4] Smits, S. I. Dalke and T. K. Cooney. The constituent equations of piezoelectric bimorphs. Sensors and Actuators A, Vol. 28, pp 41-61, (1991).

[5] De Lit, P., Agnus, J. and Chaillet, N. The constitutive equations of a piezoelectric duo-bimorph Proc. of the $5^{\text {th }}$ IEEE. International Symposium on Assembly and Task Planning, pp. 1-6. Besançon, France, July 2003.

[6] Pérez, R., Agnus, J., Clévy, C., Hubert, A. and Chaillet, N. Modelling, fabrication and validations of a high performant 2 DoF piezoactuator for micromanipulation. IEEE Mechatronics (submitted in December 2003). 


\section{FIGURES}

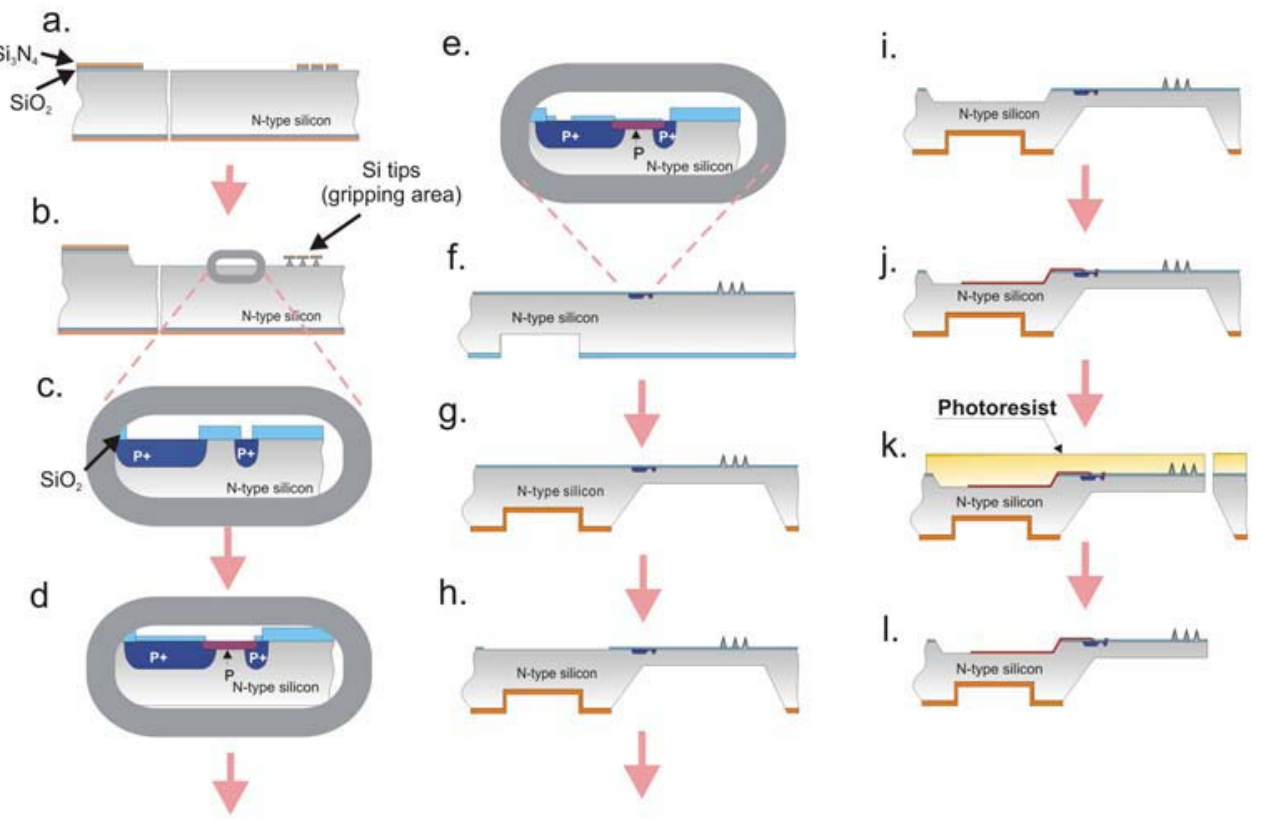

Fig. 1: The fabrication sequence a) photolithography of Si tips in the gripping area; b) KOH etching of silicon formation of tips; c) phosphorous and boron diffusions formation of diffusion paths; d)boron implantation and annealing - formation of piezoresistors; e) photolithography of contacts, f) photolithography of backside mounting cavities; g) fabrication of silicon membrane (KOH etching); h) photolithography of depression for contact pads; i) fabrication of depression for contact pads (KOH etching); j) deposition and photolithography of metal; k) photolithography of cantilevers; 1) separation of devices
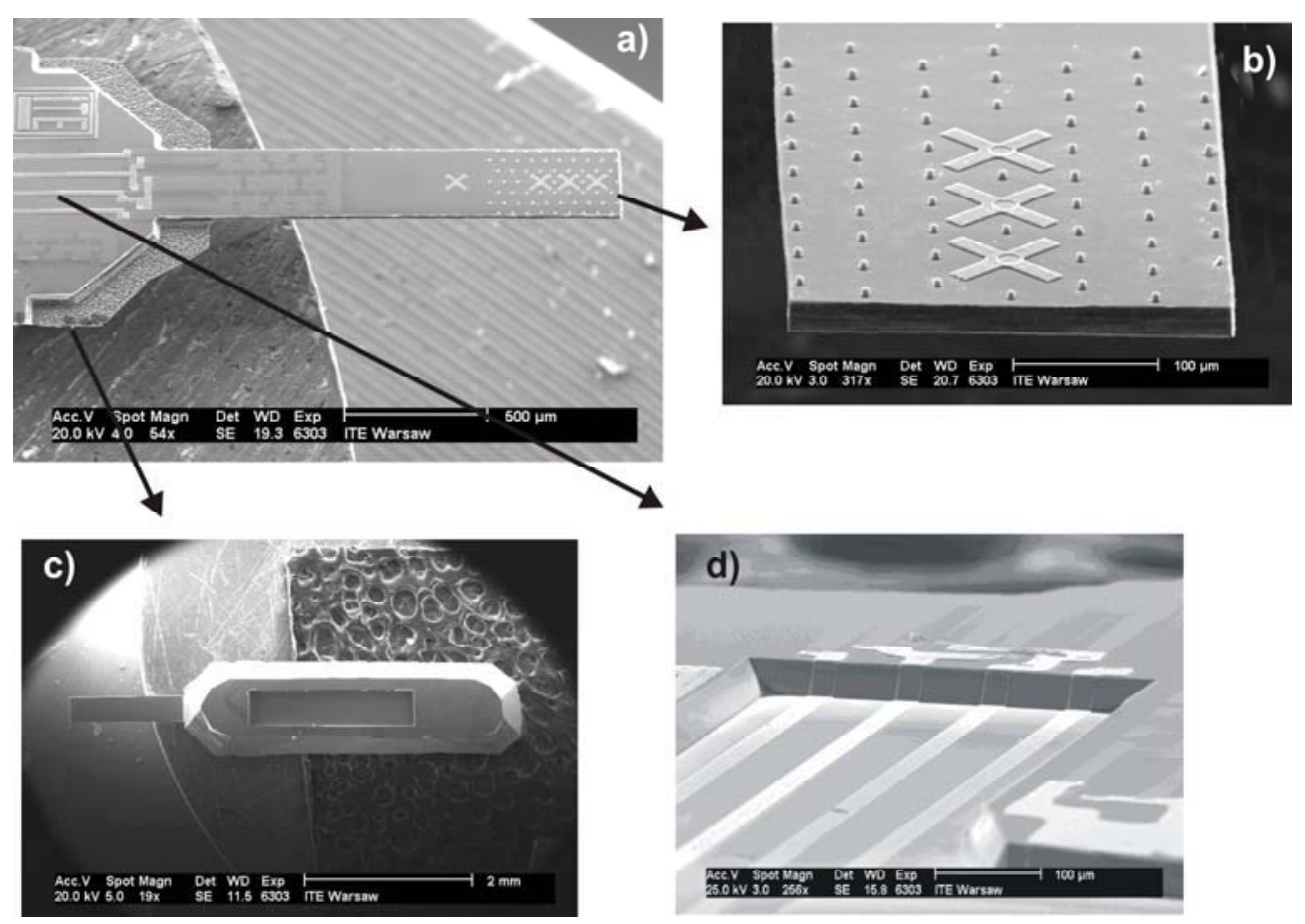

Fig. 2: SEM image of the force sensor: a) front side of the sensor with piezoresitive cantilever, b) end of the cantilever (gripping area), c) backside cavity, d) front-side depression for pads. 


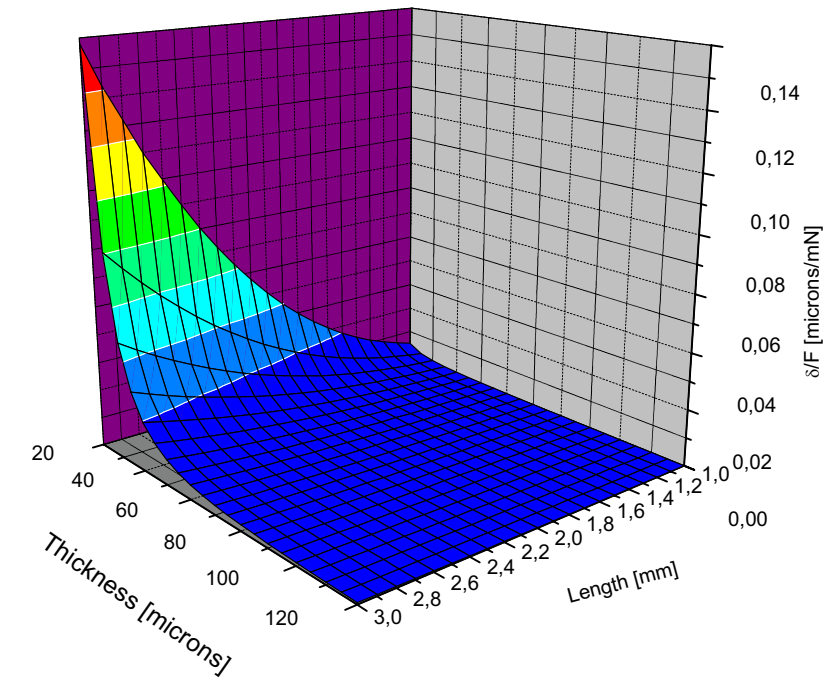

Fig. 3: Scaling of a silicon force sensor with a d/F ratio depending on thickness $t$ and length $\mathrm{L}$. Width of the silicon beam is 350 microns.

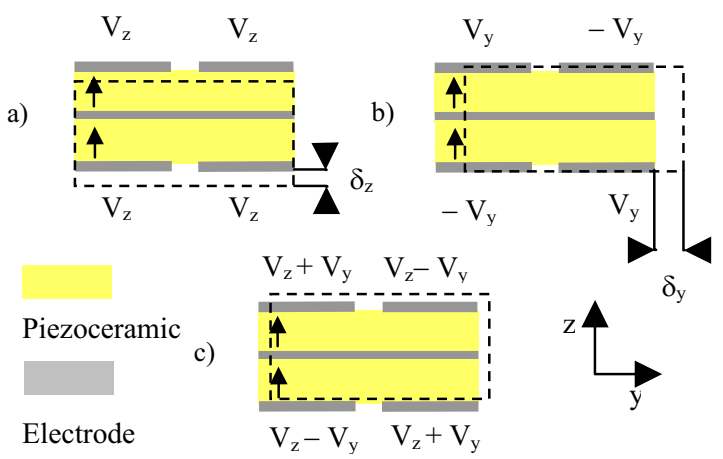

Fig 4: Working principle of the MOC with z-, y-mode and coupled y-z-mode. All three modes can be used for manipulation tasks.

\begin{tabular}{|l|r|l|}
\hline \hline$\delta_{\mathrm{y}}$ & 80 & $\mu \mathrm{m}$ \\
\hline$\delta_{\mathrm{z}}$ & 200 & $\mu \mathrm{m}$ \\
\hline $\mathrm{f}_{\mathrm{y}}$ & 1000 & $\mathrm{~Hz}$ \\
\hline $\mathrm{f}_{\mathrm{z}}$ & 400 & $\mathrm{~Hz}$ \\
\hline $\mathrm{H}$ & 25 & $\%$ \\
\hline \hline
\end{tabular}

Table 1: Working characteristics of the MOC. Displacements correspond to a voltage of $100 \mathrm{~V}$. 
a)

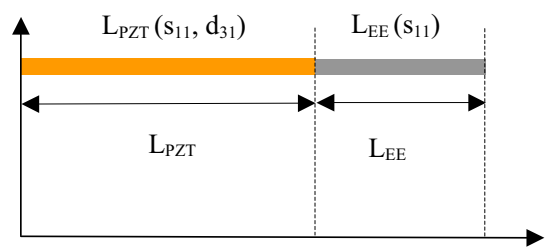

b)

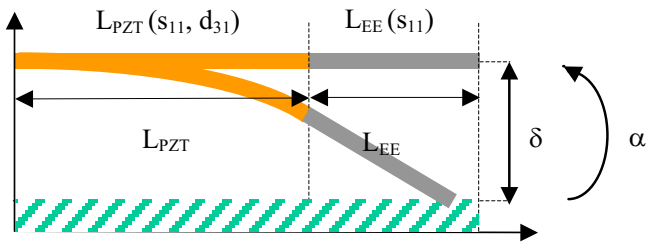

Minimum gap

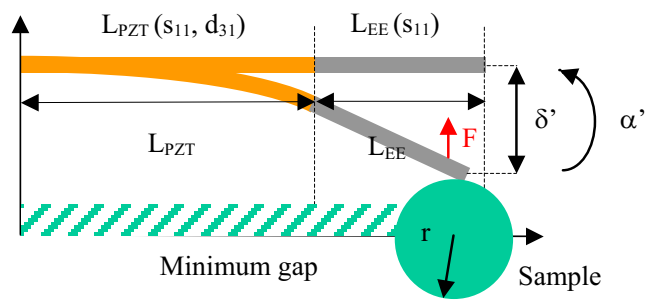

Fig. 5: Simplified model of the micromanipulator handling a sample of diameter 2 r.

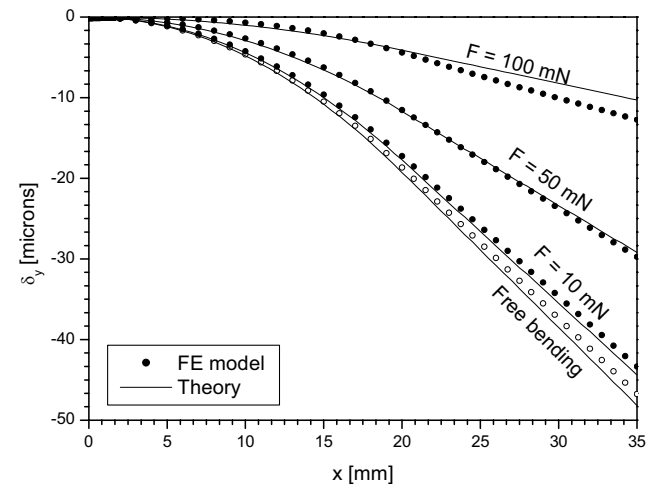

Fig. 6: Comparison of the FE results and the analytical model for the y-mode.

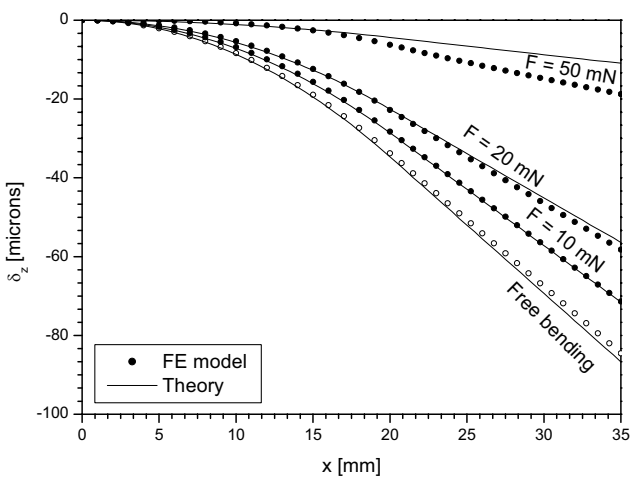

Fig. 7: Comparison of the FE results and the analytical model for the z-mode. 


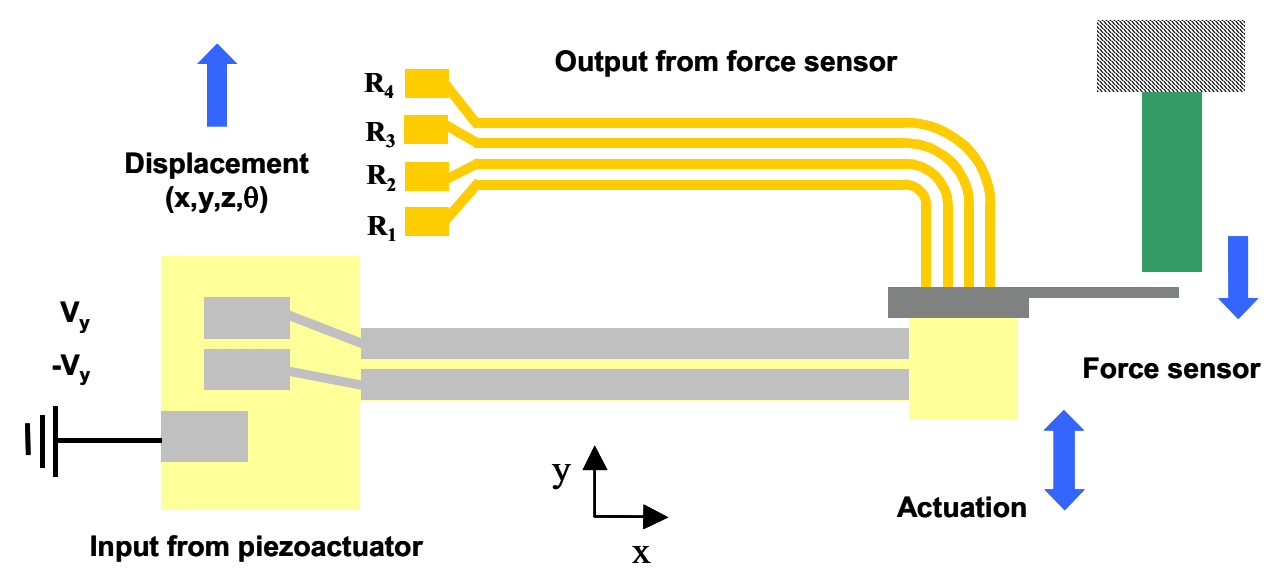

Fig. 8: Experimental set-up used to the characterisation of the integrated silicon force sensor on the piezoceramic actuator. Displacement of the whole structure has been made with a micrometric $x-y-z$ stage.

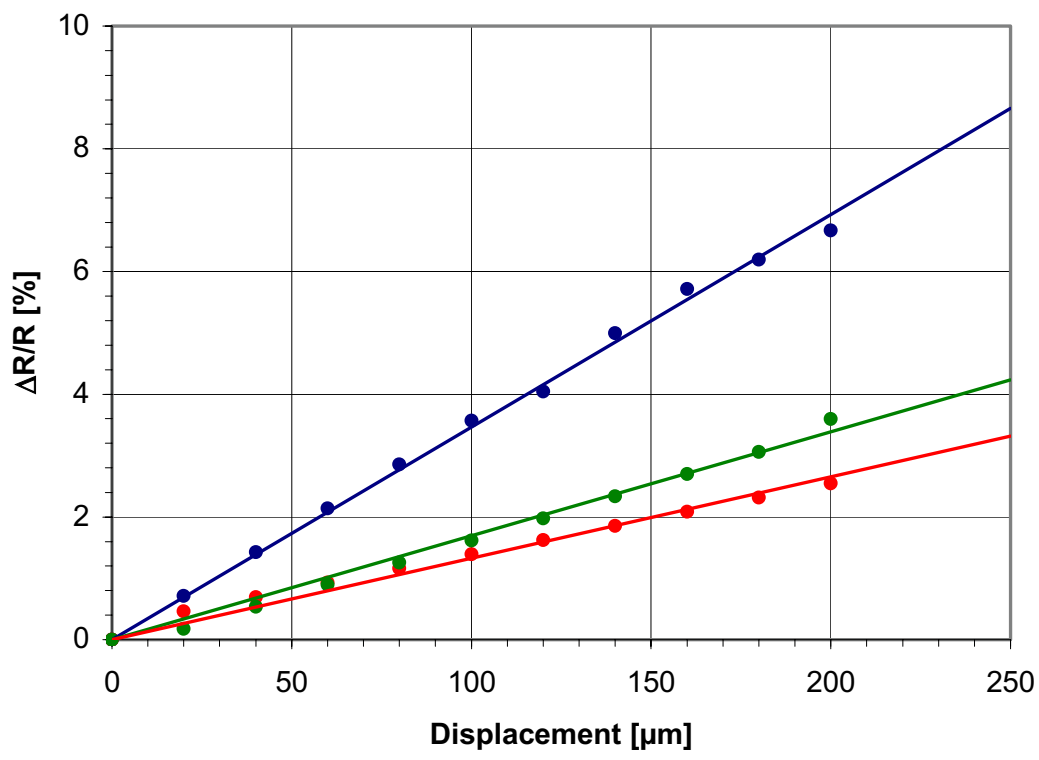

Fig. 9: Results of the characterisation tests of the piezoactuator with integrated silicon force sensor. Measurements of the resistance change versus displacement with the fitting function are shown for three different cases. 